\title{
Urgent considerations for assessment of coma in the emergency department
}

\author{
Tammam Mozher Aldarwish ${ }^{1 *}$, Talal Hussain Alolowi ${ }^{2}$, Rayan Othman Alsadiqi ${ }^{3}$, \\ Hussain Ali Al Hassan ${ }^{4}$, Abdullah Saeed Alqahtani ${ }^{5}$, Ahmed Mohammed Al Hammad \\ Wafa Mohammad Almalki ${ }^{7}$, Jawad Mubarak Al Zara ${ }^{8}$, Saud Dhafer Alshahrani', \\ Bashayer Ali Alyami ${ }^{10}$, Ahmed Hamoud Alotaibi ${ }^{11}$
}

\author{
${ }^{1}$ Department of Emergency Medicine, King Fahad General Hospital, Jeddah, Saudi Arabia \\ ${ }^{2}$ Department of Emergency Medicine, Al Madinah General Hospital, Medina, Saudi Arabia \\ ${ }^{3}$ Department of Emergency Medicine, Al Iman General Hospital, Riyadh, Saudi Arabia \\ ${ }^{4}$ College of Medicine, King Faisal University, Alahsaa, Saudi Arabia \\ ${ }^{5}$ Department of Emergency Medicine, Ahad Rufaidah General Hospital, Ahad Rufaidah, Saudi Arabia \\ ${ }^{6}$ Department of Emergency Medicine, Qatif Central Hospital, Qatif, Saudi Arabia \\ ${ }^{7}$ College of Medicine, Arab Gulf University, Manama, Bahrain \\ ${ }^{8}$ College of Medicine, Gdansk Medical University, Gdansk, Poland \\ ${ }^{9}$ College of Medicine, University of Bisha, Bisha, Saudi Arabia \\ ${ }^{10}$ College of Medicine, King Khalid University, Abha, Saudi Arabia \\ ${ }^{11}$ College of Medicine, Imam Mohammad Ibn Saud Islamic University, Riyadh, Saudi Arabia
}

Received: 09 September 2021

Accepted: 24 September 2021

\section{*Correspondence:}

Dr. Tammam Mozher Aldarwish,

E-mail: tiredness5@gmail.com

Copyright: ( ) the author(s), publisher and licensee Medip Academy. This is an open-access article distributed under the terms of the Creative Commons Attribution Non-Commercial License, which permits unrestricted non-commercial use, distribution, and reproduction in any medium, provided the original work is properly cited.

\begin{abstract}
Not speaking, closed eyes, absent response to tactile, verbal or noxious stimuli are characteristic of comatose patients. Many causes of coma have been identified and reported in the literature. However, other causes might require extensive assessment and evaluation approaches. The attending physicians start with the baseline support of life for the affected patients, followed by rapid assessment and evaluation of the etiology and pathophysiology and adequate physical examination. In addition, it requires imaging and laboratory investigations whenever needed to determine the most appropriate management plan. In this literature review, we have conducted a brief discussion about the urgent considerations for the assessment of coma in the emergency department. Many causes can attribute to the development of coma. Therefore, adequate assessment and evaluation is a key component in the management of the affected patients in the emergency department. Moreover, the management approach might differ based on the underlying etiology. Nevertheless, caring for breathing, circulation, management of the airways, and stabilization are the main steps that should be done for each comatose patient. Physical examination is also critical in such situations, where imaging or conducting laboratory tests might not be suitable for some patients. Finally, the treatment plan should be directed based on the underlying etiology of coma.
\end{abstract}

Keywords: Coma, Emergency, Management, Evaluation 


\section{INTRODUCTION}

The absence of arousal (vigilance, wakefulness), being aware of self and the surrounding environmental factors are the main characteristics of coma. ${ }^{1}$ Not speaking, closed eyes, absent response to tactile, verbal or noxious stimuli are characteristic of comatose patients. Many causes of coma have been identified and reported in the literature. However, other causes might require extensive assessment and evaluation approaches. Additionally, the therapy and diagnosis of coma should be conducted simultaneously to adequately manage the affected patients and enhance their prognosis. This can be urgently done by giving the priority to the most common causes for rapid detection of the underlying etiology. ${ }^{2}$ Besides, a brain injury should still be suspected even in cases when there is an apparent absence of signs of trauma, especially among the elderly population and patients receiving anticoagulation medications. ${ }^{3}$

Among the various conditions within the emergency department, coma has been considered a relatively rare condition in such settings, nonetheless, these patients usually require rapid interventions to prevent the development of any complications that may end up with death in critically ill patients. The attending physicians start with the baseline support of life for the affected patients, followed by rapid assessment and evaluation of the etiology, pathophysiology, adequate physical examination and imaging and laboratory investigations whenever needed to determine the most appropriate management plan. Therefore, in the current study, we aim to conduct a literature review aiming to briefly discuss the urgent considerations for the assessment of coma in the emergency department.

\section{METHODS}

This literature review is based on an extensive literature search in Medline, Cochrane, and EMBASE databases which was performed on $20^{\text {th }}$ August 2021 using the medical subject headings (MeSH) or a combination of all possible related terms. Papers discussing the assessment of coma in the emergency department were screened for relevant information, with no limitation on date, language, age of participants, or publication type.

\section{DISCUSSION}

Two main grading systems of coma have been adequately validated in the literature, including the Glasgow coma scale (GCS), and the full outline of unresponsiveness (FOUR) score that were first reported in 1974, and 2005, respectively. ${ }^{4-7}$ Both modalities assess the depth of coma, and the FOUR scores have two benefits over the GCS, including better prediction of mortality in severely ill patients, and that all of its criteria to evaluate intubated patients are reliable (Figure 1). The operational definition of a coma might be considered a reflexive, eye-closed, and non-purposeful state or absence of responsiveness to the surrounding stimuli. It has been demonstrated that the early responsibility of the physician is to determine the state and degree of responsiveness to properly determine the adequate management plan based on the patient's status and expected prognosis. For this purpose, verbal and auditory stimuli can be used to give the patient the least minimal stimuli to be evoked. Tactile stimulation can also be used to escalate the stimulation process in the regions with the largest cortical representations, like the face. Furthermore, Noxious stimulation can then be used if the patient is still unresponsive. However, the physician should be careful not to induce a significant tissue injury. Nail bed pressure, sternal rub, applying pressure to the posterior part of the mandibular ramus, or the supraorbital ridges. Opening the eyelids might also determine whether the patient has a pathological or a functional cause of coma. Arm dropping towards the patient's face is also another test that should be considered in such situations to determine which patients are conscious. Following this, initial stabilization, adequate examination, diagnosis, and treatment of the patient are required.

\begin{tabular}{|lc}
\hline Glasgow Coma Scale & Full Outline of Unresponsiveness Score \\
\hline Eye Opening Response & Eye response \\
Spontaneously, 4 & Open and tracking or blinking to command, 4 \\
To speech, 3 & Open but not tracking, 3 \\
To pain, 1 & Closed but open to loud voice, 2 \\
Best Verbal Response & Closed but open to pain, 1 \\
Oriented, 5 & Closed with pain, 0 \\
Disoriented, 5 & Motor response \\
Inappropriate words, 4 & Thumbs up, fist or peace sign, 4 \\
Incomprehensible sounds, 3 & Localizes pain, 3 \\
No response, 1 & Flexion response to pain, 2 \\
Best Motor Response & Extension response to pain, 1 \\
Obeys commands, 6 & No response to pain or myoclonic status, 0 \\
Localizes pain, 5 & Brainstem reflexes \\
Withdrawal from pain, 4 & Pupil and corneal reflexes present, 4 \\
Decorticate flexion, 3 & 1 pupil wide and fixed, 3 \\
Decerebrate extension, 2 & Pupil or corneal reflexes absent, 2 \\
No movement, 1 & Pupil and corneal reflexes absent, 1 \\
& Absent pupil, corneal and cough reflex, 0 \\
& Respiration \\
& Not intubated, regular pattern, 4 \\
& Not intubated, Cheyne-Stokes pattern, 3 \\
& Not intubated, irregular breathing, 2 \\
& Breathes above ventilator rate, 1 \\
& Breathes at ventilator rate or apnea, 0 \\
\hline
\end{tabular}

Figure 1: The GCS and FOUR score.,

\section{Stabilization and assessment considerations}

The initial stabilization procedures for comatose patients in the emergency department are similar to other situations that need surgical interventions. Securing the circulation, patent airways (giving adequate considerations to the spine), and breathing are the main points that should be initially provided for all admitted patients. Taking decisions that are related to the status of the airways of the affected patients can be difficult sometimes, and is usually dependant on several factors. For instance, it has been demonstrated that the mechanism of coma decides some important management actions based on the detected mechanism and grading of coma. For instance, it has been demonstrated that intubation should be considered for patients suffering from traumas with a GCS of $\leq 8 .{ }^{8,9}$ Besides, patients with the same grading but who suffer from a poison-induced 
coma can adequately be managed without intubation and with a minimal number of induced complications. ${ }^{10-12}$ Another important factor to consider that can determine the appropriate management plan is adequate monitoring of the underlying cause. For instance, intubation might be indicated for patients that might require computerized tomography (CT), which is usually an indicator that these patients with eventually spend some time outside the emergency department. On the other hand, intubation is not a required procedure in patients that have been indicated for acute interventions. Clinician's expectations of the prognosis and evaluation of the underlying cause is also another important factor to consider. For instance, coma secondary to alprazolam administration does not usually require intubation. On the other hand, airway interventions are usually indicated for patients that suffer from a coma that is attributable to carbamazepine administration. When comatose patients, which is attributable to trauma, have been observed to suffer from altered mental status during the initial assessment, adequate considerations should be provided to the cervical spines of these patients, especially when caring for the airways. In addition, previous studies have demonstrated that around $5 \%$ of patients in these situations will eventually have a cervical spine injury, which is usually associated with worsened prognosis and unfavorable outcomes of the managed patients. ${ }^{13,14}$

Rapid assessment of the patient should be considered to early apply the interventions and intervene against the development of any permanent complications that would deteriorate the outcomes (Figure 2). Taking full history from the patient and performing rapid physical examination should be initiated as early as possible to decide the best management approach for the patient based on the underlying etiology of coma and the status of the affected patient. Clinicians might also use laboratory and imaging studies for further evaluation of the cases and adequate diagnosis. However, these modalities should be carefully approached and they do not usually replace a comprehensive physical examination. For instance, it has been demonstrated that conducting CT is of little value when assessing the etiology of coma secondary to generalized hypoglycemia causing a non-specific neuronal dysfunction. ${ }^{15}$ On the other hand, if neuronal dysfunction was noticed secondary to trauma, performing CT is then necessary for adequate evaluation of these cases and determination of the most appropriate treatment plan. Furthermore, it has been indicated that magnetic resonance imaging modalities can also be of great importance as they can greatly provide distinct imaging of the different brain structures more than the findings that can be obtained when using CT. In cases with suspected vascular dysfunction, performing angiography has been recommended. For instance, magnetic resonance angiography has been recommended in cases of basilar artery occlusion, nevertheless, these modalities are not usually available at some healthcare centers. Therefore, other substitutes are usually used, instead. Besides, these modalities might also be limited by the cost, accessibility, and time that is required to finish the scan. Many laboratory investigations can also be urgently recommended for comatose patients to furtherly help with the assessment plan. Some of these usually include performing a complete blood picture to evaluate the presence of leukocytosis or reduced platelets count to assess infections and hemorrhage, respectively, performing an electrocardiogram for excluding toxic etiologies of coma, assessing the blood glucose level, serum electrolytes, hepatic and renal function tests. Assessment of the serum ammonia levels and arterial blood gases can also add to the diagnostic approach to adequately identify the underlying etiology and decide the best management modality. ${ }^{16}$ In another context, it has been demonstrated that performing urine drug screen testing is not favorable in these situations, as indicated by many previous investigations that such tests do not help with the process of decision making. Therefore, it should not be considered urgent approaches. ${ }^{17-20}$ Although electroencephalographic testing can aid the diagnostic approach and decision making, especially in patients with a previous significant history of brain disorders, it is not recommended for patients within the emergency department. Therefore, other alternatives and evaluation approaches are encouraged. ${ }^{21}$ A lumbar puncture can be also used. However, they should not be routinely done unless they were indicated after an adequate evaluation. ${ }^{22}$

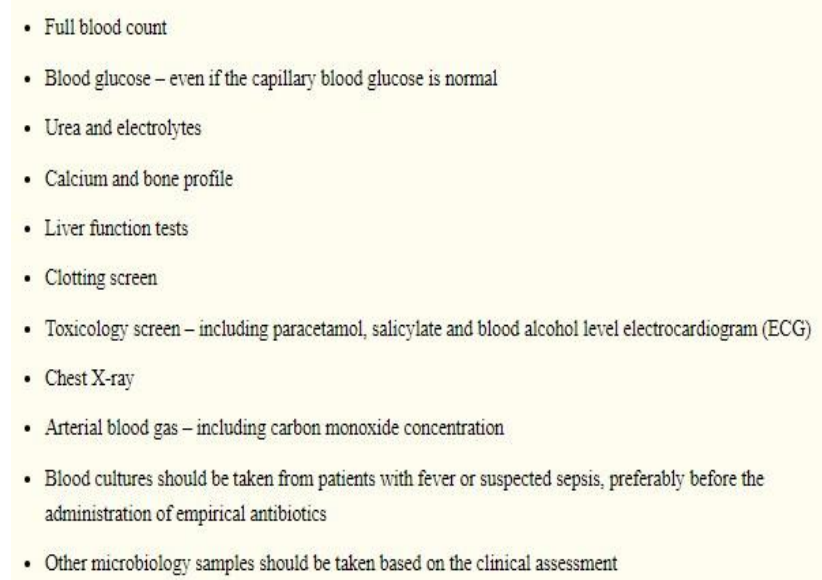

Figure 2: Early diagnostic and evaluation procedures for unconscious patients.

\section{Treatment considerations}

The treatment of comatose patients mainly depends on the underlying etiology. However, a general scheme for the management of comatose patients has been demonstrated by the different studies in the literature. Structural etiologies causing coma might have lethal or catastrophic complications and might end up with death. Therefore, detecting the underlying etiologies with adequately established diagnosis might decide whether these patients would require urgent management 
approaches or not (Figure 3 and 4). However, when the etiology has been identified, the case can be adequately managed by surgical intervention, using specific mechanical and pharmacological modalities. Moreover, consulting neurosurgical specialists is a recommended approach in such situations, especially in patients suffering from hydrocephalus and intracranial hemorrhage because the prognosis of such cases is bad and some of them might even end up with death or other serious complications. Therefore, adequate management by specialized opinions are required. Urgent assessment of patients with ischemic cardiovascular diseases is also encouraged to enhance the outcomes. A specialized team, including a neurologist, should also be involved in such situations to enhance the associated complications and enhance the outcomes of the affected patients. The team should also adequately assess whether intra-arterial or intervenous thrombolysis is suitable for these patients. Neurological dysfunctions that might occur secondary to metabolic etiologies should be considered to make sure that the patient's hemodynamics have been stabilized and intervene against the progression of any further complications. In cases when the patient suffers from respiratory insufficiency and hypoglycemia, the management plan in such situations is to try to normalize the patient's biological values, like the partial pressure carbon dioxide and oxygen, and serum glucose levels. In other situations when the patients might be suffering from hyponatremia or encephalopathy, a partial correction is the most appropriate management for these patients because it has been previously demonstrated that complete normalization might induce serious complications to these patients. ${ }^{23}$ Neuronal dysfunctions that are related to toxic events and related coma should be also adequately assessed and managed to achieve better outcomes. The most appropriate intervention that was validated for more than five decades is providing adequate support and palliative care to the affected patients. ${ }^{24}$ Assuring adequate ventilation and oxygenation, securing the airways, maintaining proper hemodynamics using intravenous fluids and vasopressors administration whenever needed. Besides, alterations to the compartmental or systemic $\mathrm{pH}$ might also be approached in such situations, which might provide advanced care and support to the affected patients by decreasing drug-related toxicity and enhancing the excretion of drugs causing toxic events. Some of these might include sodium bicarbonate administration for salicylates or tricyclic antidepressant toxicities, and administering intravenous lipid emulsion aiming at enhancing drug distribution within the body. ${ }^{25}$ Besides, it has been demonstrated that some toxin-induced states of coma might be treated by antibody-specific therapy. However, many cases have been observed with minimal or absent role against these drugs. Therefore, other management modalities should be considered in such situations to enhance the prognosis of the affected patients as early as possible. Some of the proposed antibody-based therapy include hydroxocobalamin for cyanide, fomepizole for alcohol-related toxicities, and the administration of naloxone for opioid-related toxicities.

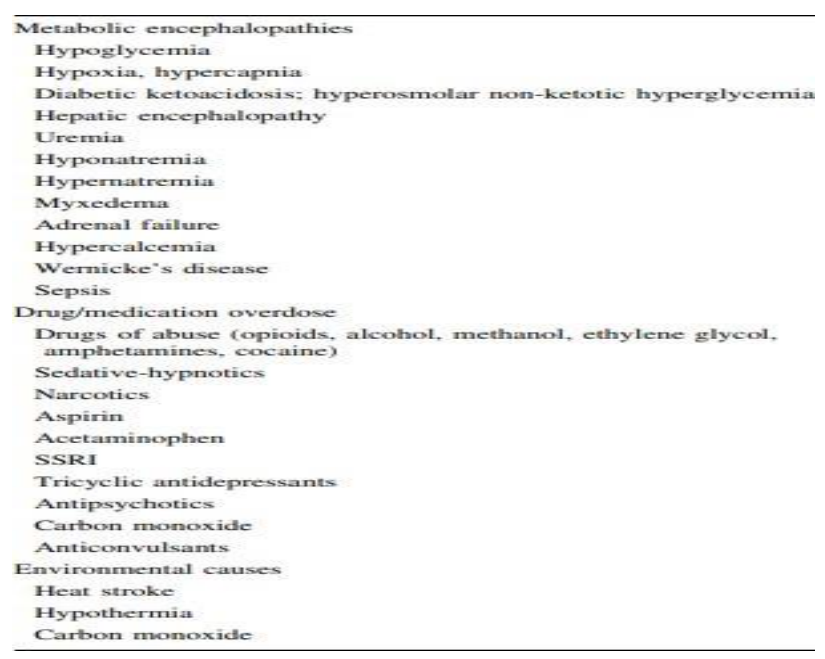

Figure 3: Toxo-metabolic causes of coma.

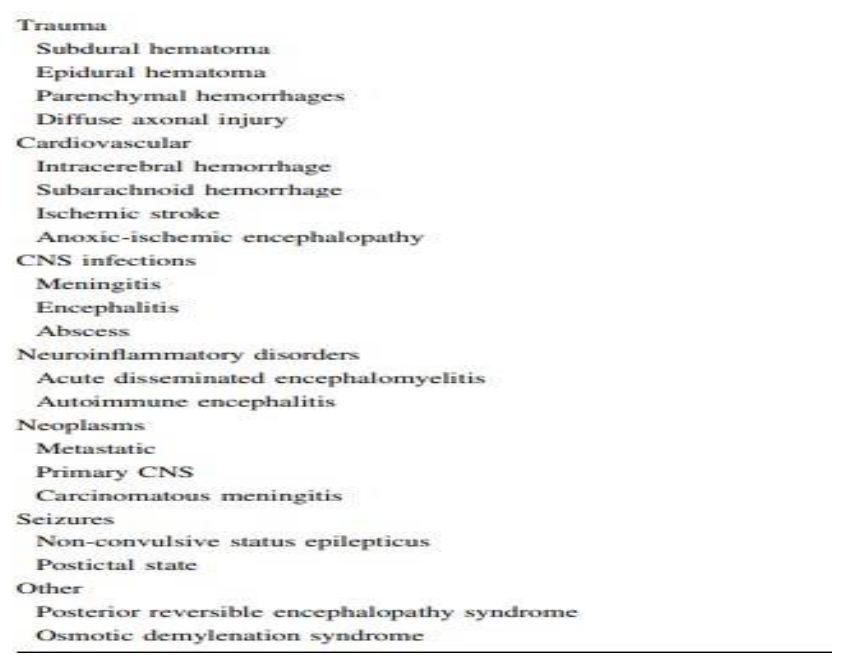

Figure 4: Neurological causes of coma.

\section{CONCLUSION}

Many causes can attribute to the development of coma. Therefore, adequate assessment and evaluation are key components in the management of the affected patients in the emergency department. The management approach might differ based on the underlying etiology. However, caring for breathing, circulation, management of the airways and stabilization are the main steps that should be done for each comatose patient. Physical examination is also critical in such situations, where imaging or conducting laboratory tests might not be suitable for some patients. Finally, the treatment plan should be directed based on the underlying etiology of coma.

Funding: No funding sources Conflict of interest: None declared

Ethical approval: Not required 


\section{REFERENCES}

1. Plum F, Posner JB. The diagnosis of stupor and coma. Contemporary neurol series. 1972;10:1-286.

2. Stevens RD, Bhardwaj A. Approach to the comatose patient. Crit Care Med. 2006;34(1):31-41.

3. Cooksley T, Rose S, Holland M. A systematic approach to the unconscious patient. Clin med. 2018;18(1):88-92.

4. Teasdale G, Jennett B. Assessment of coma and impaired consciousness. A practical scale. Lancet. 1974;2(7872):81-4.

5. Wijdicks EF, Bamlet WR, Maramattom BV, Manno EM, McClelland RL. Validation of a new coma scale: The FOUR score. Ann neurol. 2005;58(4):585-93.

6. Sadaka F, Patel D, Lakshmanan R. The FOUR score predicts outcome in patients after traumatic brain injury. Neurocrit Care. 2012;16(1):95-101.

7. Wijdicks EF, Kramer AA, Rohs T Jr. Comparison of the Full Outline of UnResponsiveness score and the Glasgow Coma Scale in predicting mortality in critically ill patients. Crit Care Med. 2015;43(2):439-44.

8. Dunham CM, Barraco RD, Clark DE. Guidelines for emergency tracheal intubation immediately after traumatic injury. J Trauma. 2003;55(1):162-79.

9. Davis DP. Prehospital intubation of brain-injured patients. Current opinion in critical care. 2008;14(2):142-8.

10. Dietze P, Horyniak D, Agius P. Effect of intubation for gamma-hydroxybutyric acid overdose on emergency department length of stay and hospital admission. Academic emergency med. 2014;21(11):1226-31.

11. Duncan R, Thakore S. Decreased Glasgow Coma Scale score does not mandate endotracheal intubation in the emergency department. J emergency med. 2009;37(4):451-5.

12. Thieu H, Bach Dat B, Nam NH. Antibiotic resistance of Helicobacter pylori infection in a children's hospital in Vietnam: prevalence and associated factors. Minerva med. 2020;111(5):498-501.

13. Schenarts PJ, Diaz J, Kaiser C, Carrillo Y, Eddy V, Morris JA Jr. Prospective comparison of admission computed tomographic scan and plain films of the upper cervical spine in trauma patients with altered mental status. J Trauma. 2001;51(4):663-68.

14. Diaz JJ Jr, Gillman C, Morris JA Jr, May AK, Carrillo YM, Guy J. Are five-view plain films of the cervical spine unreliable? A prospective evaluation in blunt trauma patients with altered mental status. J Trauma. 2003;55(4):658-63.
15. Forsberg S, Höjer J, Ludwigs U, Nyström $H$. Metabolic vs structural coma in the ED--an observational study. Am J emergency med. 2012;30(9):1986-90.

16. Cuturic M, Abramson RK. Acute hyperammonemic coma with chronic valproic acid therapy. Ann pharmacotherapy. 2005;39(12):2119-23.

17. Bertol E, Vaiano F, Borsotti M, Quercioli M, Mari F. Comparison of immunoassay screening tests and LCMS-MS for urine detection of benzodiazepines and their metabolites: results of a national proficiency test. J analytical toxicol. 2013;37(9):659-64.

18. Rengarajan A, Mullins ME. How often do falsepositive phencyclidine urine screens occur with use of common medications? Clin toxicol. 2013;51(6):493-6.

19. Brett AS. Implications of discordance between clinical impression and toxicology analysis in drug overdose. Arch internal med. 1988;148(2):437-41.

20. Bast RP, Helmer SD, Henson SR, Rogers MA, Shapiro WM, Smith RS. Limited utility of routine drug screening in trauma patients. Southern med $\mathrm{j}$. 2000;93(4):397-9.

21. Bautista RE, Godwin S, Caro D. Incorporating abbreviated EEGs in the initial workup of patients who present to the emergency room with mental status changes of unknown etiology. $\mathrm{J}$ clin neurophysiol. 2007;24(1):16-21.

22. Brouwer MC, Thwaites GE, Tunkel AR, Van de Beek D. Dilemmas in the diagnosis of acute community-acquired bacterial meningitis. Lancet. 2012;380(9854):1684-92.

23. Rafat C, Flamant M, Gaudry S, Vidal-Petiot E, Ricard JD, Dreyfuss D. Hyponatremia in the intensive care unit: How to avoid a Zugzwang situation? Ann intensive care. 2015;5(1):39.

24. Clemmesen C, Nilsson E. Therapeutic trends in the treatment of barbiturate poisoning. The Scandinavian method. Clin pharmacol therapeutics. 1961;2:220-29.

25. Ozcan MS, Weinberg G. Intravenous lipid emulsion for the treatment of drug toxicity. $\mathrm{J}$ intensive care med. 2014;29(2):59-70.

Cite this article as: Aldarwish TM, Alolowi TH, Alsadiqi RO, Al Hassan HA, Alqahtani AS, Al Hammad AM et al. Urgent considerations for assessment of coma in the emergency department. Int J Community Med Public Health 2021;8:5489-93. 